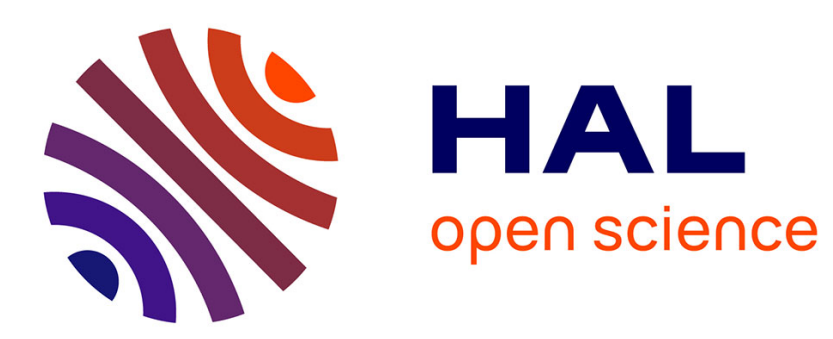

\title{
A Semi-Analytical Model for Delay/Doppler Altimetry and Its Estimation Algorithm
}

Abderrahim Halimi, Corinne Mailhes, Jean-Yves Tourneret, Pierre Thibault, François Boy

\section{- To cite this version:}

Abderrahim Halimi, Corinne Mailhes, Jean-Yves Tourneret, Pierre Thibault, François Boy. A SemiAnalytical Model for Delay/Doppler Altimetry and Its Estimation Algorithm. IEEE Transactions on Geoscience and Remote Sensing, 2014, vol. 52 ( $\mathrm{n}^{\circ}$ 7), pp. 4248-4258. 10.1109/TGRS.2013.2280595 . hal-01057698

\section{HAL Id: hal-01057698 https://hal.science/hal-01057698}

Submitted on 25 Aug 2014

HAL is a multi-disciplinary open access archive for the deposit and dissemination of scientific research documents, whether they are published or not. The documents may come from teaching and research institutions in France or abroad, or from public or private research centers.
L'archive ouverte pluridisciplinaire HAL, est destinée au dépôt et à la diffusion de documents scientifiques de niveau recherche, publiés ou non, émanant des établissements d'enseignement et de recherche français ou étrangers, des laboratoires publics ou privés. 


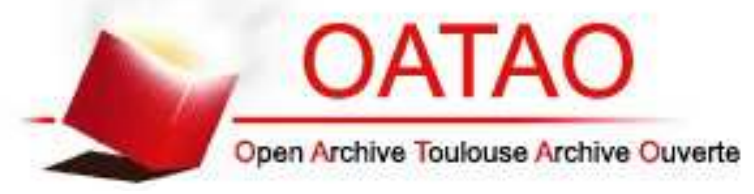

\section{Open Archive TOULOUSE Archive Ouverte (OATAO)}

OATAO is an open access repository that collects the work of Toulouse researchers and makes it freely available over the web where possible.

This is an author-deposited version published in : http://oatao.univ-toulouse.fr/ Eprints ID : 11930

To link to this article : doi:10.1109/TGRS.2013.2280595

URL : http://dx.doi.org/10.1109/TGRS.2013.2280595

To cite this version : Halimi, Abderrahim and Mailhes, Corinne and Tourneret, Jean-Yves and Thibault, Pierre and Boy, François A SemiAnalytical Model for Delay/Doppler Altimetry and Its Estimation Algorithm. (2014) IEEE Transactions on Geoscience and Remote Sensing, vol. 52 ( $\mathrm{n}^{\circ}$ 7). pp. 4248-4258. ISSN 0196-2892

Any correspondance concerning this service should be sent to the repository administrator: staff-oatao@ listes-diff.inp-toulouse.fr 


\title{
A Semi-Analytical Model for Delay/Doppler Altimetry and Its Estimation Algorithm
}

\author{
Abderrahim Halimi, Student Member, IEEE, Corinne Mailhes, Member, IEEE, \\ Jean-Yves Tourneret, Senior Member, IEEE, Pierre Thibaut, and Francois Boy
}

\begin{abstract}
The concept of delay/Doppler (DD) altimetry (DDA) has been under study since the mid-1990s, aiming at reducing the measurement noise and increasing the along-track resolution in comparison with the conventional pulse-limited altimetry. This paper introduces a new model for the mean backscattered power waveform acquired by a radar altimeter operating in synthetic aperture radar mode, as well as an associated least squares (LS) estimation algorithm. As in conventional altimetry (CA), the mean power can be expressed as the convolution of three terms: the flat surface impulse response (FSIR), the probability density function of the heights of the specular scatterers, and the time/frequency point target response of the radar. An important contribution of this paper is to derive an analytical formula for the FSIR associated with DDA. This analytical formula is obtained for a circular antenna pattern, no mispointing, no vertical speed effect, and a uniform scattering. The double convolution defining the mean echo power can then be computed numerically, resulting in a 2-D semi-analytical model called the DD map (DDM). This DDM depends on three altimetric parameters: the epoch, the sea surface wave height, and the amplitude. A multi-look model is obtained by summing all the reflected echoes from the same along-track surface location of interest after applying appropriate delay compensation (range migration) to align the DDM on the same reference. The second contribution of this paper concerns the estimation of the parameters associated with the multi-look semi-analytical model. An LS approach is investigated by means of the Levenberg-Marquardt algorithm. Simulations conducted on simulated altimetric waveforms allow the performance of the proposed estimation algorithm to be appreciated. The analysis of Cryosat-2 waveforms shows an improvement in parameter estimation when compared to the CA.
\end{abstract}

Index Terms-Altimetry, Cryosat, delay/Doppler (DD) map (DDM), least squares (LS) estimation, synthetic aperture radar (SAR) altimetry.

A. Halimi, C. Mailhes, and J.-Y. Tourneret are with the Institut de Recherche en Informatique de Toulouse (UMR 5505)/École Nationale Supérieure d'Électronique, d'Électrotechnique, d'Informatique, d'Hydraulique et des Télécommunications, Institut National Polytechnique de Toulouse/ Telecommunications for Space and Aeronautics, University of Toulouse, 31071 Toulouse, France (e-mail: Abderrahim.Halimi@enseeiht.fr; Corinne. Mailhes@enseeiht.fr; Jean-Yves.Tourneret@enseeiht.fr).

P. Thibaut is with Collecte Localisation Satellites, 31520 Ramonville-SaintAgne, France (e-mail: pthibaut@cls.fr).

F. Boy is with the Centre National d'Études Spatiales, 31401 Toulouse, France (e-mail: Francois.Boy@cnes.fr).

Digital Object Identifier 10.1109/TGRS.2013.2280595

\section{INTRODUCTION}

$\mathbf{F}$ OR more than 20 years, conventional altimeters like Topex, Poseidon-2, or Poseidon-3 have been delivering waveforms which are used to estimate many parameters such as the range between the satellite and the observed scene. The theoretical model for this conventional altimetric waveform is provided by a convolution between three terms that are the flat surface impulse response (FSIR), the probability density function (PDF) of the heights of the specular scatterers, and the point target response (PTR) of the radar [1], [2]. Several analytical formulations for the FSIR have been proposed in the literature, leading to the Brown model [1] and to more elaborated altimetric waveform models [3]-[5]. Many other studies have been devoted to improve the oceanic analytical model in order to get better estimates of the geophysical parameters. This improvement has been obtained by using different PDF and PTR formulations [2], [6], [7]. Recently, a great effort has also been devoted to process coastal waveforms in order to move the altimetric measurements closer to the coast [8]-[11].

The delay/Doppler (DD) altimetry (DDA) proposed in [12] fits into this logic of measurement improvement and has two main objectives. The first one is to reduce the measurement noise by increasing the number of observations (looks) which provide better geophysical parameter estimates. The second one is to increase the along-track resolution which allows the measurements to remain valid until a distance of about $300 \mathrm{~m}$ from the coast [while it was about $10 \mathrm{~km}$ for conventional altimetry (CA)]. All of these advantages have led to consider DDA in many current and future satellite missions. The first satellite exploiting DDA is the Cryosat-2 satellite which has on board a synthetic aperture interferometric radar altimeter (SIRAL) instrument that includes a DDA mode. Other future missions including DDA are Sentinel-3, Jason Continuité de Service (Jason-CS), and Surface Water Ocean Topography (SWOT), which show the importance of this new technology.

DDA requires coherent correlation between pulses [12], which is obtained by transmitting pulses with a high pulserepetition frequency (PRF). For instance, the SIRAL transmits bursts with a frequency of about $85 \mathrm{~Hz}$ [13]. Each burst contains 64 coherent pulses (transmitted at a PRF of $18182 \mathrm{~Hz}$ ) which are processed to obtain the DD map (DDM), as shown in Fig. 1. Note that transmitting 64 coherent pulses results in 64 spectral Doppler beams in the DDM, as illustrated in Fig. 1. The exploitation of the DDA oceanic information is based on the analysis of the reflected oceanic waveform called multilook echo and obtained by applying Doppler processing (slant 

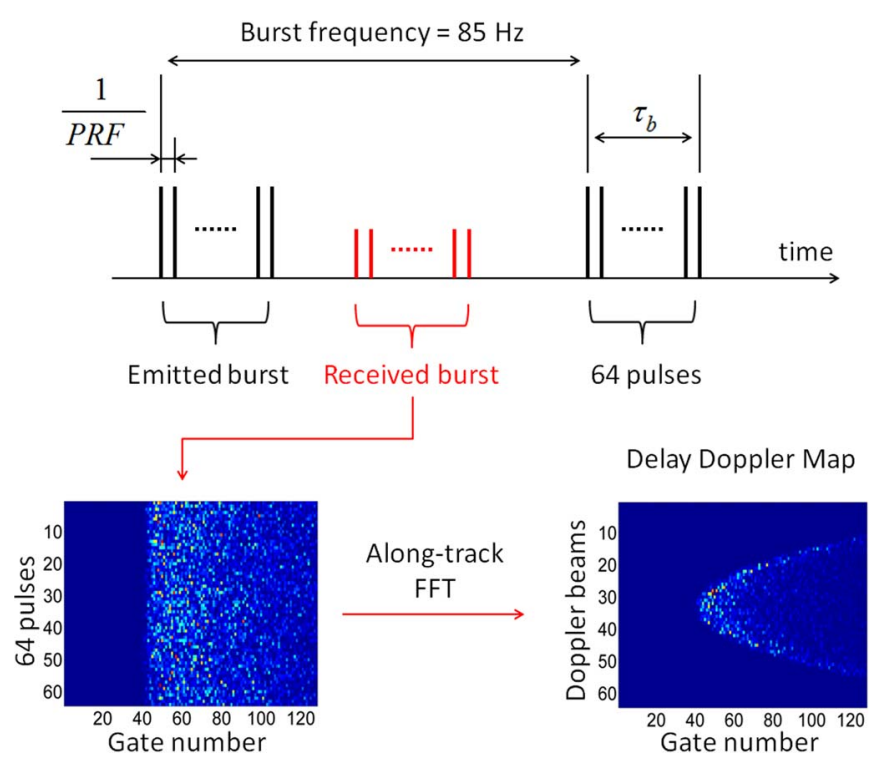

Fig. 1. Configuration of a DD altimeter and construction of a DDM.

range correction and multi-looking) to the DDM. This multilook waveform has a shape that is different from a CA echo, which requires developing a new altimetric signal model. Many studies have been conducted by different teams for achieving this goal. For instance, numerical models for DD waveforms have been proposed in [14] and [15], whereas other models were developed in the Synthetic aperture radar Altimetry MOde Studies and Applications (SAMOSA) project [16], [17].

The first contribution of this paper is the derivation of a new model for DDA. An analytical model for the FSIR is studied based on a geometrical approach. The proposed FSIR model includes Earth curvature and considers a circular antenna pattern, no mispointing, and a Gaussian approximation for the antenna gain as in [1]. The resulting analytical expression of the FSIR is numerically convolved with the PDF of the sea wave height and the PTR of the radar. This yields the mean power of a DDA waveform which depends on three parameters: the epoch $\tau$, the significant wave height (SWH), and the amplitude $P_{u}$.

The second contribution of this paper is to propose and validate an algorithm for estimating the parameters of the proposed DD semi-analytical model. Many different algorithms have been investigated to estimate the parameters of CA waveforms. These algorithms are, for instance, based on the maximum-likelihood principle [6], [18] or on least squares (LS) techniques [19], [20]. This paper proposes to estimate the geophysical altimetric parameters by an LS approach based on the Levenberg-Marquardt algorithm. The performance of the estimated parameters is analyzed in different scenarios including different noise configurations. Moreover, the evaluation of the estimated parameters, on simulated and real Cryosat-2 data, provides a quantitative measure of the benefits of DDA when compared to CA.

This paper is organized as follows. Section II presents the transition from the conventional altimetric model to the proposed DD semi-analytical model. The proposed LS estimation procedure is then introduced in Section III. Section IV validates

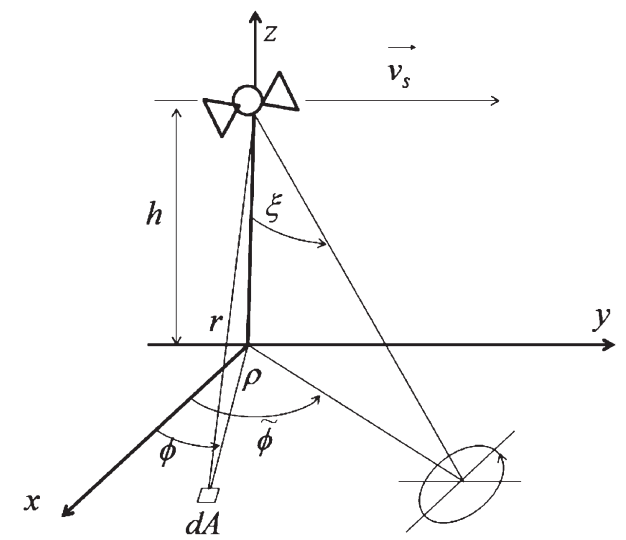

Fig. 2. Geometry used for computing the FSIR.

the proposed model and algorithm with simulated data. The analysis of results associated with real Cryosat- 2 waveforms is presented in Section V. Conclusions and future work are finally reported in Section VI.

\section{Semi-Analytical Model for Delay/Doppler ALTIMETRY}

This section first describes the CA model and then introduces the proposed semi-analytical model for DD waveforms. The multi-look processing and the corruption of the waveforms by speckle noise are also described.

\section{A. Conventional Altimetry}

In $\mathrm{CA}$, the mean power $P(t)$ is expressed as the convolution of three terms: the FSIR, the PDF of the heights of the specular scatterers, and the PTR of the radar

$$
P(t)=\operatorname{FSIR}(t) * \operatorname{PDF}(t) * \mathrm{PTR}_{T}(t)
$$

where $t$ is the two-way incremental ranging time, i.e., $t=$ $t^{\prime}-(2 h / c)$, with $t^{\prime}$ the travel time of the echo from the instant of transmission, $h$ the altitude of the satellite, and $c$ the speed of light. The following sections describe the three terms in (1).

1) Flat Surface Impulse Response: The FSIR only depends on time and is obtained by integrating over the illuminated area of the surface as follows [1]:

$$
\operatorname{FSIR}\left(t^{\prime}\right)=\frac{\lambda^{2}}{(4 \pi)^{3} L_{p}} \int_{\mathbb{R}^{+} \times[0,2 \pi[} \frac{\delta\left(t^{\prime}-\frac{2 r}{c}\right) G^{2}(\rho, \phi) \sigma^{0}}{r^{4}} \rho d \rho d \phi
$$

where $\rho$ and $\phi$ are the radius and the angle of the polar coordinates, respectively, $L_{p}$ is the two-way propagation loss, $\lambda$ is the wavelength, $G$ is the power gain of the radar antenna, $\delta(t)$ is the delta function, $\sigma^{0}$ is the backscatter coefficient of the surface, and $r=\sqrt{\rho^{2}+h^{2}}$ is the range between the satellite and the observed surface (see Fig. 2). The integral with respect to $\rho$ in (2) can be expressed in closed form when considering a constant value of $\sigma^{0}$, an antenna without mispointing 


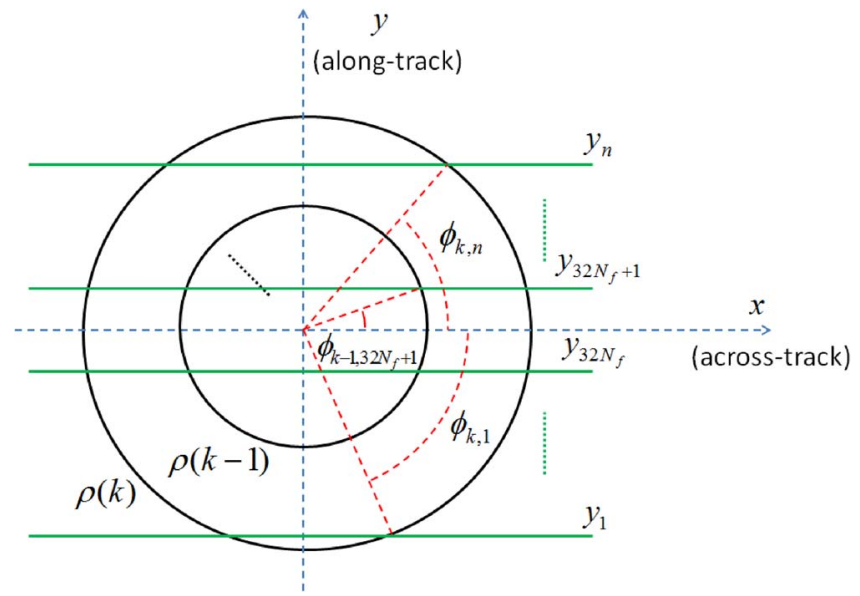

Fig. 3. Circles of propagation and Doppler beams. In CA, the FSIR is obtained by integrating over the propagation circles. In DDA, the FSIR is obtained by integrating the energy in the intersection between the propagation circles and the Doppler beams.

angles with respect to the $z$ - and $x$-axes $\left(\xi=0^{\circ}\right.$ and $\tilde{\phi}=0^{\circ}$ in Fig. 2), and the same gain antenna as in [1], i.e., a Gaussian approximation and a circular antenna pattern. The FSIR is then given by

$$
\operatorname{FSIR}(t)=\frac{P_{u}}{2 \pi}\left(1+\frac{c t}{2 h}\right)^{-3} U(t) \int_{0}^{2 \pi} \exp \left(-\frac{4 c t}{\gamma h}\right) d \phi
$$

where $\gamma=\frac{1}{2 \ln 2} \sin ^{2} \theta_{3 \mathrm{~dB}}$ is an antenna beamwidth parameter, $\theta_{3 \mathrm{~dB}}$ is the half-power antenna beamwidth, $P_{u}=$ $\left(\lambda^{2} G_{0}^{2} c \sigma^{0}\right) /\left[4(4 \pi)^{2} L_{p} h^{3}\right]$ is the waveform amplitude, $G_{0}$ is the antenna power gain at boresight, and $U(\cdot)$ denotes the Heaviside function $(U(t)=1$ if $t \geq 0$ and $U(t)=0$ if $t<0)$. Equation (3) shows that $\operatorname{FSIR}(t)$ is obtained by integrating an appropriate function on a circle whose radius $\rho(t)$ depends on time, i.e., for each time instant $t$, we have a given radius (see Fig. 3). This radius increases with time since $\rho\left(t^{\prime}\right)=$ $\sqrt{\left(t^{\prime} c / 2\right)^{2}-h^{2}}$ which reduces to $\rho(t) \simeq \sqrt{h c t}$ when considering the approximation $(c t / h) \ll 1$ (valid for spaceborne altimetry [1]). Note also that, in CA, we integrate all along the circle of radius $\rho$ (since $\phi \in[0,2 \pi]$ ) without having a distinction between across-track and along-track directions (axes $x$ and $y$ in Fig. 3, respectively). The conventional FSIR is finally given by [1], [5]

$$
\operatorname{FSIR}(t)=P_{u} \exp \left(-\frac{4 c t}{\gamma h}\right) U(t)
$$

where $(1+(c t / 2 h))^{-3}$ has been approximated by 1 as in [1] (since $(c t / h) \ll 1)$.

2) PDF of the Heights of the Specular Scatterers: The PDF of the specular points is generally approximated by a Gaussian density whose standard deviation (STD) is related to the average SWH [1], [5]

$$
\operatorname{PDF}(t)=\frac{1}{\sqrt{2 \pi} \sigma_{s}} \exp \left(-\frac{t^{2}}{2 \sigma_{s}^{2}}\right)
$$

with $\sigma_{s}=\mathrm{SWH} /(2 c)$.

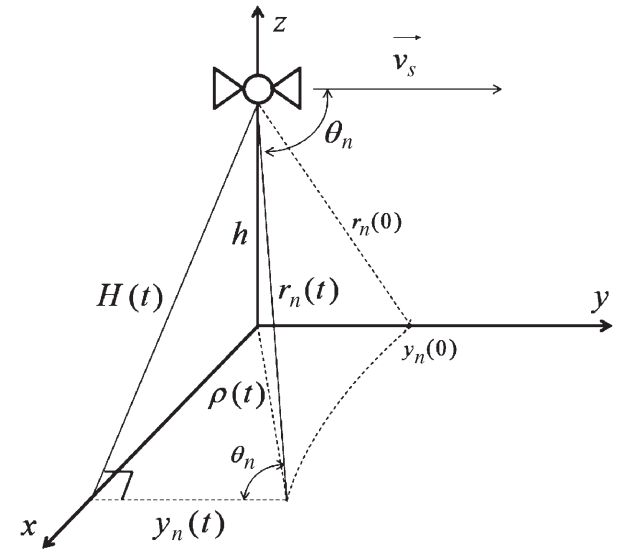

Fig. 4. Doppler beam geometry.

3) Radar System PTR: The radar PTR is generally expressed as a squared cardinal sine as follows [5]:

$$
\operatorname{PTR}_{T}(t)=\left|\frac{\sin \left(\pi \frac{t}{T}\right)}{\pi \frac{t}{T}}\right|^{2}
$$

where $T=1 / B$ is the sampling period and $B$ is the reception bandwidth of the altimeter.

\section{B. Delay/Doppler Altimetry}

As in CA, the mean power $P(t, f)$ of a DD echo can be expressed as the convolution of three terms: the FSIR, the PDF, and the time/frequency PTR [21], [22]. However, unlike the signal $P(t)$ in (1), the obtained signal $P(t, f)$ depends on time and Doppler frequency as follows:

$$
P(t, f)=\operatorname{FSIR}(t, f) * \operatorname{PDF}(t) * \operatorname{PTR}(t, f)
$$

where $f$ denotes the Doppler frequency. The PDF is the same as that in (5), and the two other terms are introduced in the following.

1) Flat Surface Impulse Response: The DDA is pulse limited across-track and beam limited along-track as first observed by Raney in [12]. It was proposed in order to increase the alongtrack resolution by considering the Doppler effect resulting from the satellite velocity. Indeed, the $n$th Doppler frequency $f_{n}$ is expressed by

$$
f_{n}=\frac{2}{\lambda} \frac{\vec{r} \cdot \vec{v}_{s}}{|\vec{r}|}=\frac{2 v_{s}}{\lambda} \cos \left(\theta_{n}\right)
$$

where $\vec{v}_{s}$ is the satellite velocity and $\theta_{n}$ is shown in Fig. 4. This figure also shows that

$$
\cos \left(\theta_{n}\right)=\frac{y_{n}(t)}{r_{n}(t)}=\frac{y_{n}(t)}{\sqrt{\rho^{2}(t)+h^{2}}}, \quad \text { for } t \geq 0
$$

where $y_{n}(t)$ represents the coordinate of the $n$th along-track beam. Combining (8) and (9) leads to the following expression of $y_{n}(t)$ as a function of $t$ and $f_{n}$ :

$$
y_{n}(t)=\left(\frac{\lambda f_{n}}{2 v_{s}}\right) \sqrt{\rho^{2}(t)+h^{2}} .
$$


This equation clearly shows how the coordinate of the alongtrack beam depends on time. An approximation of (10) is obtained by considering $\rho(t) \ll h$ which is a valid assumption for near-vertical small-angle geometry as explained in [12] (see [23] for more details about this approximation). The simplified width of the Doppler beam is then given by [12]

$$
y_{n}=\frac{h \lambda}{2 v_{s}} f_{n}
$$

with $f_{n}=\left(n-32 N_{f}-0.5\right) F / N_{f}$, for $n \in 1, \ldots, 64 N_{f}$, where $F$ is the frequency resolution obtained from the burst length $\tau_{b}=1 / F$ (see Fig. 1 ) and $N_{f}$ is the frequency oversampling factor. This equation shows that the along-track direction (axis $y$ ) can be divided into rectangular beams corresponding to different Doppler frequencies displayed in Fig. 3.

Fig. 3 also shows that the computation of the FSIR for DDA is obtained by integrating $\phi$ into rectangular beams defined by fixed coordinates $y_{n}$ and $y_{n+1}$ (we will consider the timeindependent Doppler coordinate given in (11) in the rest of this paper). Straightforward computations show that the angles associated with $y_{n}$ and $y_{n+1}$ are defined by

$$
\begin{aligned}
\phi_{t, n} & =\operatorname{Re}\left[\operatorname{atan}\left(\frac{y_{n}}{\sqrt{\rho^{2}(t)-y_{n}^{2}}}\right)\right] \\
\phi_{t, n+1} & =\operatorname{Re}\left[\operatorname{atan}\left(\frac{y_{n+1}}{\sqrt{\rho^{2}(t)-y_{n+1}^{2}}}\right)\right]
\end{aligned}
$$

where $\operatorname{atan}(\cdot)$ is the inverse tangent function and $\operatorname{Re}(x)$ denotes the real part of the complex number $x$. As a consequence, the DDA FSIR can be written ${ }^{1}$

$$
\operatorname{FSIR}(t, n)=\frac{P_{u}}{2 \pi} U(t) \int_{\mathcal{D}_{t, n}} \exp \left(-\frac{4 c t}{\gamma h}\right) d \phi
$$

where $\mathcal{D}_{t, n}=\left[\phi_{t, n}, \phi_{t, n+1}\right] \cup\left[\pi-\phi_{t, n+1}, \pi-\phi_{t, n}\right]$. Note that the conventional FSIR can be obtained by considering the angles $\phi_{t, n+1}=\pi / 2$ and $\phi_{t, n}=-\pi / 2$ in $\mathcal{D}_{t, n}$. This means that the conventional FSIR given in (4) can also be obtained by summing the signals of all the Doppler beams before range migration, i.e., by summing the DDM rows (see Fig. 6(b)bottom-as an example of DDM). As a consequence, (13) leads to the following analytical expression of the FSIR:

$$
\operatorname{FSIR}(t, n)=\frac{P_{u}}{\pi} \exp \left(-\frac{4 c t}{\gamma h}\right)\left(\phi_{t, n+1}-\phi_{t, n}\right) U(t)
$$

for $n=1, \ldots, 64 N_{f}$. Note that one has to divide the time $t$ in (4) and (14) by the curvature factor $\alpha=1+(h / R)=1.11$, where $R=6378137 \mathrm{~m}$ is the Earth radius, to account for the Earth curvature (see [23], [25], and [26] for more details about Earth curvature).

2) Radar System PTR: The radar system PTR is composed of temporal and Doppler frequency dimensions. In this paper,

\footnotetext{
${ }^{1}$ A related approach assuming a rectangular shape for the compressed pulse and a rectangular antenna pattern was investigated in [24].
}

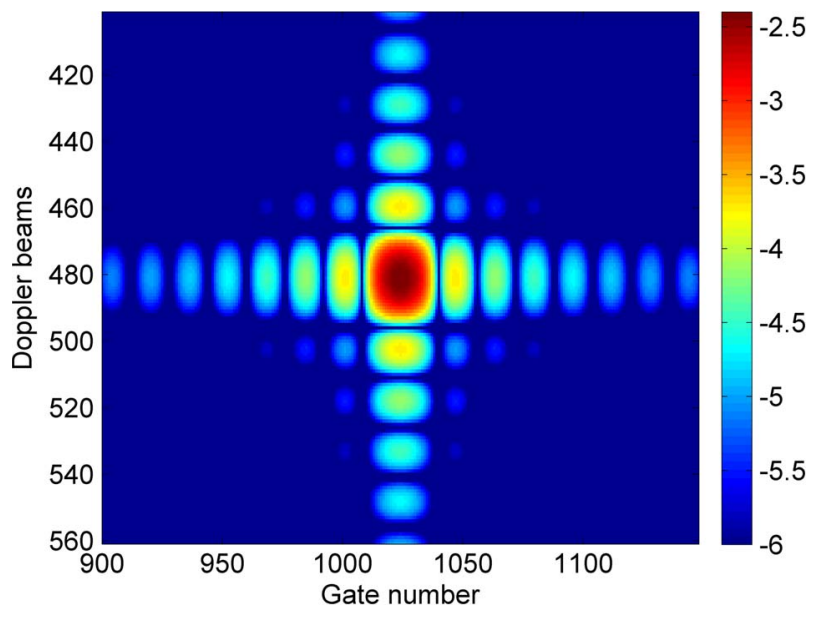

Fig. 5. Logarithm of the oversampled 2-D radar system PTR $(\log [\operatorname{PTR}(t, f)])$.

we assume that $\operatorname{PTR}(t, f)$ is the multiplication between a temporal function $\mathrm{PTR}_{T}(t)$ (corresponding to the radar PTR) and a frequency function $\operatorname{PTR}_{F}(f)$ (resulting from the Doppler processing). This assumption can be justified by recent results available in the literature [21], [22], [27] or by a comparison with the measured Cryosat-2 PTR (see [23] for more details). The temporal PTR is provided in (6), whereas $\operatorname{PTR}_{F}(f)$ can be approximated accurately by the following squared sine cardinal function:

$$
\operatorname{PTR}_{F}(f)=\left|\frac{\sin \left(\pi \frac{f}{F}\right)}{\pi \frac{f}{F}}\right|^{2} .
$$

The resulting PTR is then given by

$$
\operatorname{PTR}(t, f)=\operatorname{PTR}_{T}(t) \operatorname{PTR}_{F}(f)
$$

which is displayed in Fig. 5. It is interesting to note that another PTR could be used without modifying significantly the proposed approach (e.g., PTR $(t, f)$ might be obtained from real measurements). Indeed, the PTR will be convolved numerically with the analytical FSIR derived in this paper and the PDF defined in (5).

\section{Reflected Power}

The reflected DDA power $P(t, f)$ (resp. $P(t)$ for CA) is obtained by a numerical computation of the double convolution (7) [resp. (1)] between the analytical expressions (14), (5), and (16) [resp. (4), (5), and (6)]. This convolution is conducted after oversampling the analytical functions to better represent the cardinal sines. Appropriate temporal and frequency oversampling factors have been determined by cross-validation, yielding $N_{t}=16$ and $N_{f}=15$. The resulting oversampled signal is first delayed by the epoch $\tau$ and then undersampled to obtain the required $64 \times 128 \mathrm{DDM}$. The proposed model (7) is semi-analytical in the sense that an analytical formulation is proposed for the FSIR but that the double convolution in (7) is computed numerically. Note that the proposed semi-analytical 


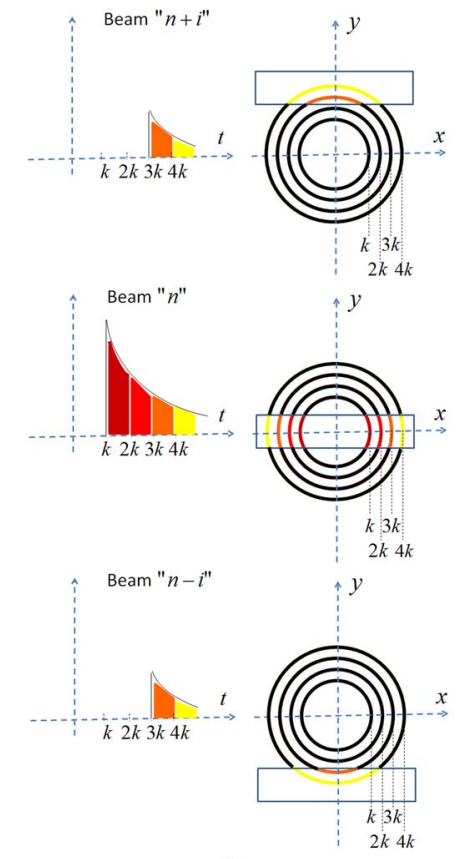

(a)
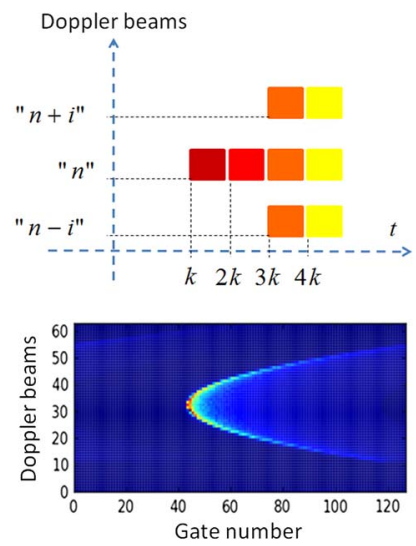

(b)
Fig. 6. Construction of the DDM.

model might be modified by introducing a measured $\operatorname{PTR}(t, f)$ and/or a PDF different from (5).

\section{Multi-looking}

Section II-B derived an analytical model for the $\operatorname{FSIR}(t, f)$ which is convolved by $\operatorname{PDF}(t)$ and $\operatorname{PTR}(t, f)$ to compute the reflected power $P(t, f)$. We also showed previously that each time instant $t$ is related to a circle of radius $\rho(t)$ while each Doppler frequency is related to a rectangular along-track beam. Fig. 6 summarizes the construction of a DDM. The signal of a given beam is obtained by summing the energies of all scatterers belonging to this beam. For instance, the energy of the signal corresponding to time instant " $k$ " and Doppler beam " $n$ " is obtained by summing the energies of all scatterers belonging to the intersection of the circle of radius $\rho(k)$ with the rectangular nadir beam " $n$." Note that the rises of the reflected powers in the different Doppler beams occur at different time instants (according to Fig. 6, the rise occurs at time instant $k$ for the nadir beam, at time instant $3 k$ for beams " $n+i$ " and " $n-i$," etc.). This time shift is related to the range between the satellite and each Doppler beam. Fig. 6(b) shows an example of a DDM obtained by the proposed model. The parabolic shape of this waveform results from the time shifts between the different beams. The multi-looking process aims at gathering all the reflected energies from a single beam. For that, we first have to compensate the time differences between the different beams in order to have signals rising at the same time instant $k$. This procedure is called delay compensation [12] or range migration. The delay of each beam $\delta r_{n}$ is obtained by the difference between the modulus of the position vector $r_{n}=\sqrt{h^{2}+y_{n}^{2}}$ (range between the satellite and the Doppler beam $n$ ) and the minimum satellite-surface distance $h$ [12]

$$
\delta r_{n}=r_{n}-h=\sqrt{h^{2}+y_{n}^{2}}-h .
$$
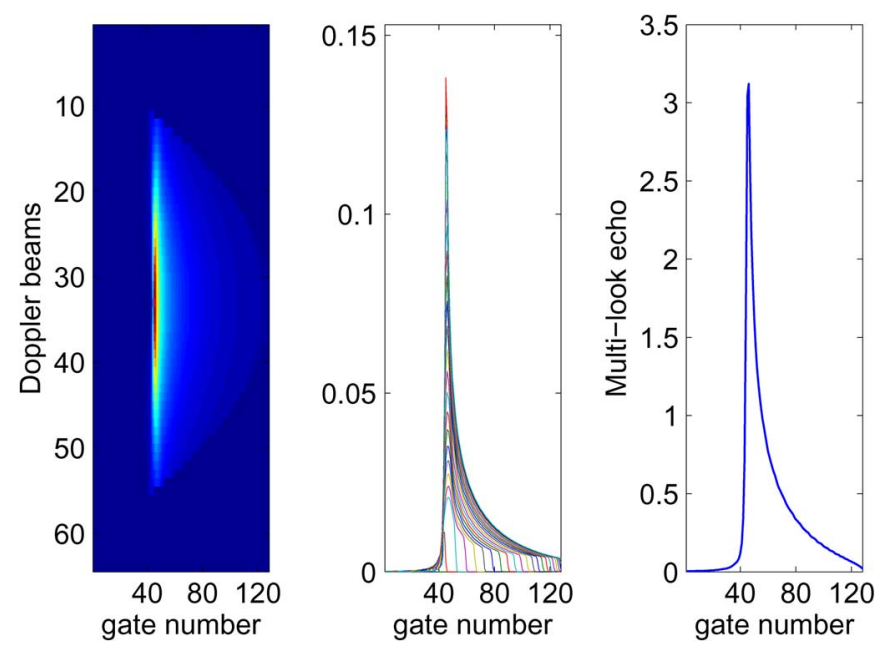

Fig. 7. (Left) DDM after delay compensation, (middle) migrated signals for all Doppler beams, and (right) the corresponding multi-look waveform.

Note that (17) can be simplified (as proposed in [12]) by considering $y_{n} \ll h$ as follows:

$$
\delta r_{n}=h \sqrt{1+\left(\frac{y_{n}}{h}\right)^{2}}-h \simeq \frac{y_{n}^{2}}{2 h}=\frac{h \lambda^{2}}{8 v_{s}^{2}} f_{n}^{2} .
$$

Note also that the Earth curvature can be considered by introducing a factor $\alpha$, yielding [12]

$$
\delta r_{n}=\sqrt{h^{2}+\alpha y_{n}^{2}}-h \simeq \alpha \frac{h \lambda^{2}}{8 v_{s}^{2}} f_{n}^{2}
$$

After delay compensation, the signals associated with the Doppler beams are summed to obtain the multi-look waveform as follows [22] (see Fig. 7):

$$
s(t)=\sum_{n=1}^{64} P\left(t-\delta t_{n}, f_{n}\right)
$$

where $\delta t_{n}=2 \delta r_{n} / c$ is the delay compensation expressed in seconds. This result is obtained by assuming that each ground Doppler beam is observed by 64 different Doppler beams where each of them results from averaging $L$ observations (see also the next section). Note that the procedure is quite different for real waveforms where we have to collect the reflected energies of different bursts. For example, the selected scene's beam may reflect energy coming from a nadir beam (beam \#33) for the burst $i_{1}$, from beam \#34 for the burst $i_{2}$, etc. Note that this stacking procedure aims at reducing the noise effect and that it assumes that the geophysical parameters of the selected beam do not change from one burst to another.

An example of the resulting DDA vector $s=\left(s_{1}, \ldots, s_{K}\right)^{T}$, with $K=104$ samples, is shown in Fig. 8 and compared with the CA echo. The DD echo has a peaky shape around the epoch $\tau$ because of delay compensation. This peaky shape was first quantified in [28] as characteristic of a beam-limited altimeter.

\section{E. Speckle Noise}

In order to generate realistic data similar to Cryosat- 2 echoes, the DDM has to be corrupted by speckle noise. Following the 


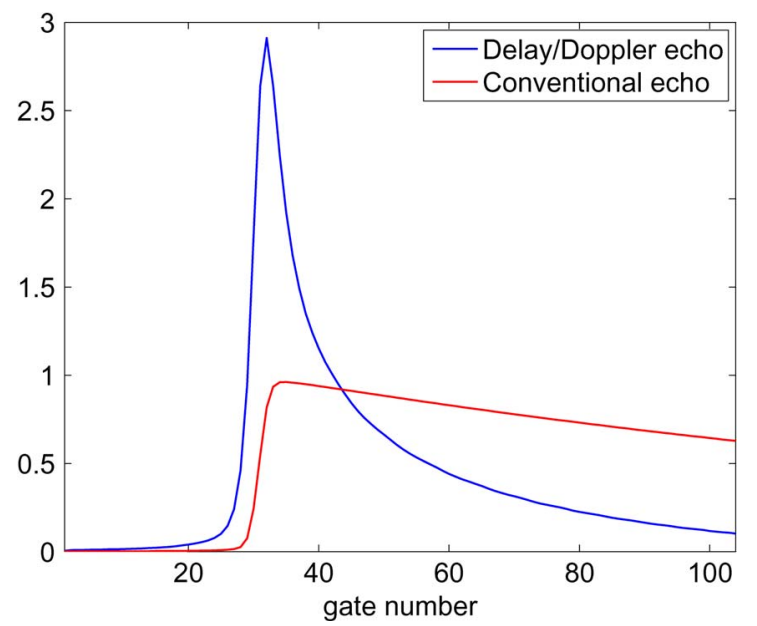

Fig. 8. DD and conventional echoes for the same altimetric parameters $\left(P_{u}=\right.$ $1, \tau=31$ gates, and $\mathrm{SWH}=2 \mathrm{~m}$ ).

works in [29], a multiplicative speckle noise is applied to the DDM, leading to ${ }^{2}$

$$
y(t)=\sum_{n=1}^{64} P\left(t-\delta t_{n}, f_{n}\right) q\left(t-\delta t_{n}, n\right)
$$

where $q\left(t-\delta t_{n}, n\right)$ is a random variable distributed according to a gamma distribution $\mathcal{G}(L, 1 / L)$ (see [32, p. 87] for the definition of the gamma distribution) and $L$ is the number of bursts observing each Doppler beam ( $L=4$ in our simulations).

\section{PARAmeter Estimation}

\section{A. Estimation Algorithm}

This paper proposes to estimate the parameters of the multilook waveform by using an LS procedure (as for CA [19], [20]). The altimetric waveform $\boldsymbol{y}=\left(y_{1}, \ldots, y_{K}\right)^{T}$ is a noisy version of $s=\left(s_{1}, \ldots, s_{K}\right)^{T}$ which depends on the parameter vector $\boldsymbol{\theta}=\left(\theta_{1}, \theta_{2}, \theta_{3}\right)^{T}=\left(\mathrm{SWH}, P_{u}, \tau\right)^{T}$ (the estimation is done under the assumption that $\xi=0^{\circ}$ in both conventional and DD models). The LS method consists of estimating the unknown parameter vector $\boldsymbol{\theta}$ as follows:

$$
\underset{\boldsymbol{\theta}}{\arg \min } \boldsymbol{G}(\boldsymbol{\theta})=\underset{\boldsymbol{\theta}}{\arg \min } \frac{1}{2} \sum_{k=1}^{K} g_{k}^{2}(\boldsymbol{\theta})
$$

where $g_{k}(\boldsymbol{\theta})=y_{k}-s_{k}(\boldsymbol{\theta})$ is a vector of residues. Since $g_{k}(\boldsymbol{\theta})$ is a complicated nonlinear function of SWH and $\tau$, the optimization problem (22) does not admit a closed-form expression. In this paper, we propose to solve (22) using a numerical optimization method based on the Levenberg-Marquardt algorithm [33]. The parameter update of the iterative LevenbergMarquardt algorithm is defined by $\boldsymbol{\theta}^{(i+1)}=\boldsymbol{\theta}^{(i)}+\boldsymbol{e}^{(i)}$, where $\boldsymbol{\theta}^{(i)}$ is the estimate of $\boldsymbol{\theta}$ at the $i$ th iteration. The choice of $\boldsymbol{e}^{(i)}$

${ }^{2}$ In [29], a single look is assumed to follow an exponential distribution. Moreover, and as mentioned in [30] and [31], each Doppler beam is observed by $L$ bursts (denoted as $N_{\text {bin }}$ in [30] and [31]). Thus, the signal of each beam results from the averaging of $L$ observations. It results that the noise corrupting each beam follows a gamma distribution $\mathcal{G}(L, 1 / L)$. is based on a Taylor expansion (at the first order) of $\boldsymbol{g}$ in the neighborhood of $\boldsymbol{\theta}^{(i)}$

$$
\boldsymbol{g}\left(\boldsymbol{\theta}^{(i)}+\boldsymbol{e}^{(i)}\right) \simeq \boldsymbol{l}\left(\boldsymbol{e}^{(i)}\right)=\boldsymbol{g}\left(\boldsymbol{\theta}^{(i)}\right)+\boldsymbol{J}\left(\boldsymbol{\theta}^{(i)}\right) \boldsymbol{e}^{(i)}
$$

where $\boldsymbol{J}(\boldsymbol{\theta})=\left[\boldsymbol{J}_{1}(\boldsymbol{\theta}), \boldsymbol{J}_{2}(\boldsymbol{\theta}), \boldsymbol{J}_{3}(\boldsymbol{\theta})\right]=\left[\left(\partial \boldsymbol{g}(\boldsymbol{\theta}) / \partial \theta_{1}\right)\right.$, $\left.\left(\partial \boldsymbol{g}(\boldsymbol{\theta}) / \partial \theta_{2}\right),\left(\partial \boldsymbol{g}(\boldsymbol{\theta}) / \partial \theta_{3}\right)\right]$ is a $K \times 3$ matrix representing the gradient of $\boldsymbol{g}$. After replacing (23) in (22) (and removing notation $(i)$ for brevity), the following result is obtained:

$$
\begin{aligned}
\boldsymbol{G}(\boldsymbol{\theta}+\boldsymbol{e}) \simeq \boldsymbol{L}(\boldsymbol{e}) & =\frac{1}{2} \boldsymbol{l}(\boldsymbol{e})^{T} \boldsymbol{l}(\boldsymbol{e}) \\
& =\boldsymbol{G}(\boldsymbol{\theta})+\boldsymbol{e}^{T} \boldsymbol{J}(\boldsymbol{\theta})^{T} \boldsymbol{g}+\frac{1}{2} \boldsymbol{e}^{T} \boldsymbol{J}(\boldsymbol{\theta})^{T} \boldsymbol{J}(\boldsymbol{\theta}) \boldsymbol{e}
\end{aligned}
$$

The descent direction $e$ is then obtained by minimizing $\boldsymbol{L}(\boldsymbol{e})$. By setting to zero the derivative $\boldsymbol{L}^{\prime}(\boldsymbol{e})=\boldsymbol{J}(\boldsymbol{\theta})^{T} \boldsymbol{g}+$ $\boldsymbol{J}(\boldsymbol{\theta})^{T} \boldsymbol{J}(\boldsymbol{\theta}) \boldsymbol{e}$, we obtain

$$
\boldsymbol{J}(\boldsymbol{\theta})^{T} \boldsymbol{J}(\boldsymbol{\theta}) \boldsymbol{e}=-\boldsymbol{J}(\boldsymbol{\theta})^{T} \boldsymbol{g} .
$$

This relation is the basis of the Gauss-Newton recursion [33], [34]. Levenberg and Marquardt proposed to add a regularization parameter $\mu$ in (25), leading to

$$
\left[\boldsymbol{J}(\boldsymbol{\theta})^{T} \boldsymbol{J}(\boldsymbol{\theta})+\mu \boldsymbol{I}_{3}\right] \boldsymbol{e}=-\boldsymbol{J}(\boldsymbol{\theta})^{T} \boldsymbol{g}
$$

where $I_{3}$ is the $3 \times 3$ identity matrix. Note that the parameter $\mu$ controls the convergence speed of the algorithm. Note also that the derivatives appearing in $\boldsymbol{J}(\boldsymbol{\theta})$ can be computed numerically by finite difference as follows:

$$
\boldsymbol{J}_{i}(\boldsymbol{\theta})=-\frac{\partial \boldsymbol{s}(\boldsymbol{\theta})}{\partial \theta_{i}} \simeq-\frac{\boldsymbol{s}\left(\theta_{i}+\Delta \theta_{i}\right)-\boldsymbol{s}\left(\theta_{i}\right)}{\Delta \theta_{i}}
$$

with $\Delta \boldsymbol{\theta}=\left(\Delta \mathrm{SWH}, \Delta \tau, \Delta P_{u}\right)^{T}$. In our simulations, we have chosen $\Delta \boldsymbol{\theta}=(0.05 \mathrm{~m}, 0.02 \text { gate }, 0.05)^{T}$.

\section{B. Estimation Performance}

This section introduces the criteria used to evaluate the quality of the estimators resulting from the proposed model. The quality of the estimation for simulated waveforms can be measured by comparing the estimated and true parameters by using the root-mean-square error (RMSE)

$$
\operatorname{RMSE}\left(\theta_{i}\right)=\sqrt{\frac{1}{m} \sum_{\ell=1}^{m}\left[\theta_{i}-\hat{\theta}_{i}(\ell)\right]^{2}}, \quad i=1, \ldots, 3
$$

where $\theta_{i}$ is the true parameter, $\hat{\theta}_{i}(\ell)$ is the estimated parameter for the $\ell$ th waveform, and $m$ is the number of simulated waveforms. The bias and the STD of the estimator given by

$$
\begin{aligned}
\operatorname{Bias}\left(\theta_{i}\right) & =\frac{1}{m} \sum_{\ell=1}^{m} \hat{\theta}_{i}(\ell)-\theta_{i}=\overline{\theta_{i}}-\theta_{i} \\
\operatorname{STD}\left(\theta_{i}\right) & =\sqrt{\frac{1}{m} \sum_{\ell=1}^{m}\left[\hat{\theta}_{i}(\ell)-\overline{\theta_{i}}\right]^{2}}
\end{aligned}
$$




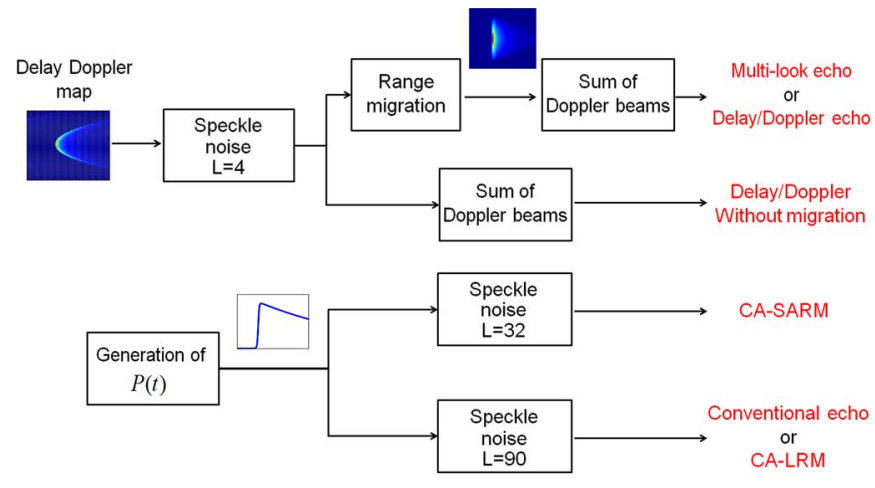

Fig. 9. Construction of the observed echoes and related terminology.

can also be used to better analyze the obtained results. In the case of real waveforms, the estimated DD parameters will be compared to the estimated CA parameters.

\section{Results For Simulated Data}

This section first describes how simulated echoes have been generated. The behavior of the proposed DD model as a function of the Doppler frequency is then analyzed. The effect of range migration on the performance of the LS estimator is finally investigated. The estimation performances of DDA and $\mathrm{CA}$ are also compared in order to illustrate the expected improvement of the DD mode (as shown in [14] and [35] for a simulated scene and in [21] for another Doppler model).

\section{A. Simulation Scenario}

This section describes how Cryosat-2 echoes have been generated and introduces the denominations of the related simulated echoes. The Cryosat- 2 altimeter called SIRAL presents three modes that are the low-resolution mode (LRM), the synthetic aperture radar mode (SARM), and the synthetic aperture radar interferometric mode (SARInM) [13]. The data of the LRM are used to generate CA echoes (also denoted by CALRM echoes), while those of SARM provide DD echoes. However, as the two modes operate separately, the collected data do not result from the same scene and cannot be used to compare the same scenario. Hence, the data of SARM are also used to generate conventional echoes called in this paper CA-SARM for conventional altimetric echoes from SARM. ${ }^{3}$ However, the resulting echoes are affected by a level of noise that is higher than that for CA-LRM echoes. Indeed, the observed CA echoes are corrupted by a speckle noise resulting from the incoherent summations of $L_{c}=90$ consecutive echoes for the Poseidon-3 altimeter [39]. The CA-SARM results from averaging approximately 32 uncorrelated echoes (the other correlated echoes will not reduce significantly the noise level), inducing a noise increasing factor of $\sqrt{3}$ between the CA and CA-SARM echoes [40]. Fig. 9 summarizes the different steps performed to obtain the considered simulated echoes

\footnotetext{
${ }^{3}$ These echoes are known under different names: LRM-like [22], pseudoLRM [36]-[38], or reduced SAR [37], [38]. The denomination CA-SARM has been chosen for clarity.
}

TABLE I

Simulation PARAMETERS

\begin{tabular}{|c|l|}
\hline Parameter & Value \\
\hline Frequency & $13.575 \mathrm{GHz}$ \\
Wavelength $(\lambda)$ & $2.21 \mathrm{~cm}$ \\
Bandwidth $(\mathrm{B})$ & $320 \mathrm{MHz}$ \\
Altitude $(h)$ & $730 \mathrm{~km}$ \\
Burst repetition frequency (BRF) & $85 \mathrm{~Hz}$ \\
Pulse repetition frequency (PRF) & $18182 \mathrm{~Hz}$ \\
$3 \mathrm{~dB}$ antenna beam width $\left(\theta_{3 \mathrm{~dB}}\right)$ & $1.1388 \mathrm{degrees}$ \\
Velocity $\left(v_{s}\right)$ & $7000 \mathrm{~m} / \mathrm{s}$ \\
Pulses per burst & 64 pulses \\
Burst length $\left(\tau_{b}\right)$ & $3.5 \mathrm{~ms}$ \\
Doppler beam width & $327 \mathrm{~m}$ \\
\hline
\end{tabular}
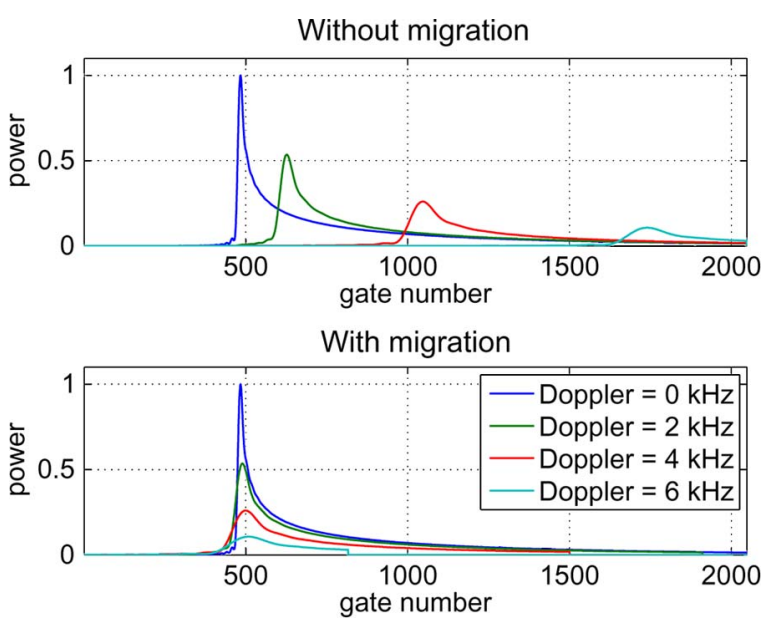

Fig. 10. Echoes for different Doppler frequencies (0, 2, 4, and $6 \mathrm{kHz})$. (Top) Without range migration and (bottom) with range migration. The temporal scale has been oversampled by a factor of $N_{t}=16$.

and their denominations in the rest of this paper, i.e., multilook (or DD), DD without migration, CA (or CA-LRM), and CA-SARM echoes.

\section{B. Model Analysis}

This section analyzes the behavior of the reflected power as a function of the Doppler frequency. An example of simulation scenario corresponding to the altimetric parameters $P_{u}=1$, $\mathrm{SWH}=0 \mathrm{~m}$, and $\tau=31$ gates is summarized in Table I. Fig. 10 shows the corresponding altimetric echoes (normalized by the maximum of the nadir echo) for different Doppler frequencies $(0,2,4$, and $6 \mathrm{kHz})$. As expected, the higher power occurs at nadir, i.e., $f=0 \mathrm{~Hz}$. This figure also shows that the echo broadens as the frequency increases, which can be explained as follows. The Doppler frequency is proportional to the along-track distance [see (11)]. As a consequence, the high frequencies correspond to far Doppler beams (from nadir) that intersect the large propagation circles. However, propagation circles have an increasing radius and a narrowing width for increasing time [41]. This means that the Doppler beams far from nadir intersect a lot of propagation circles (each circle corresponds to a time instant), and thus, the reflected echoes spread over a lot of range gates. Fig. 10 (bottom) shows an example of DD echoes obtained after range migration (this figure is similar to [29, Fig. 7]). Note that the leading edge of the multi-look echo, obtained by summing the migrated echoes, is 

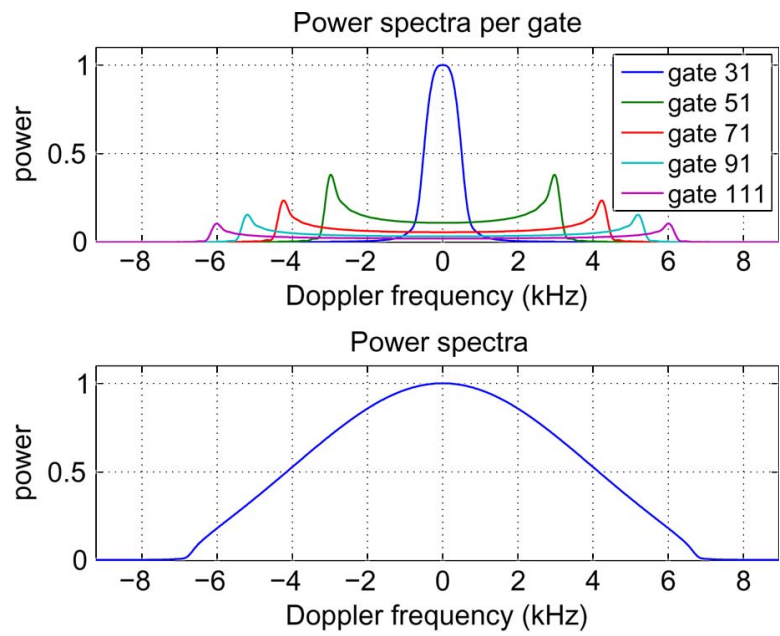

Fig. 11. (Top) Reflected power versus Doppler frequency for different gate numbers; (bottom) Doppler spectra after summing the powers associated with each Doppler frequency [sum of the columns of Fig. 7 (left)]. The frequency scale has been oversampled by a factor of $N_{f}=15$.

directly affected by the high-Doppler-frequency echoes because of their large shape and slower leading edge. Considering echoes associated with different time gates (gates 31, 51, 71, 91, and 111), Fig. 11 (top) shows a decrease of the power according to the Doppler frequency which is due to the weighting of the power by the Gaussian antenna gain [14]. This figure also shows a symmetrical shape of the echoes with respect to the zero Doppler frequency which is due to the absence of mispointing angle $\xi=0^{\circ}$ (note that the situation can be very different in the presence of mispointing as shown in [41]). These results are confirmed in Fig. 11 (bottom), which shows the Doppler spectra resulting from the summation of the powers associated with the different Doppler frequencies.

\section{Importance of Range Migration}

This section is interested in analyzing the effect of range migration on the quality of the estimated parameters. Parameter RMSEs obtained with and without range migration (with the same noise level, i.e., $L=4$ ) are shown in Fig. 12 versus SWH (additional results versus $\tau$ and $P_{u}$ are available in [23]). These RMSEs have been obtained using $m=1000$ simulated waveforms [see (28)]. The parameters SWH and $\tau$ are better estimated by considering migrated DD echoes since the errors on $\mathrm{SWH}$ and $\tau$ are reduced by $\simeq 30$ and $\simeq 6 \mathrm{~cm}$, respectively. However, the estimation of $P_{u}$ is slightly better without migration because the echo is broader and its amplitude is less sensitive to noise.

Fig. 12 also shows the RMSEs when estimating CA echoes. The DD RMSEs for $\tau$ and SWH (the blue curves in Fig. 12) are lower than those obtained with CA (the black curves in Fig. 12) which shows the interest of using the Doppler mode. However, one can notice that CA provides better results for RMSE (SWH) for very small values of SWH (this result was also observed in [22]). Note that some simulation results available in [23] (and not presented here for brevity) have shown that the proposed estimator is unbiased and thus that the RMSEs are very close to the STDs.
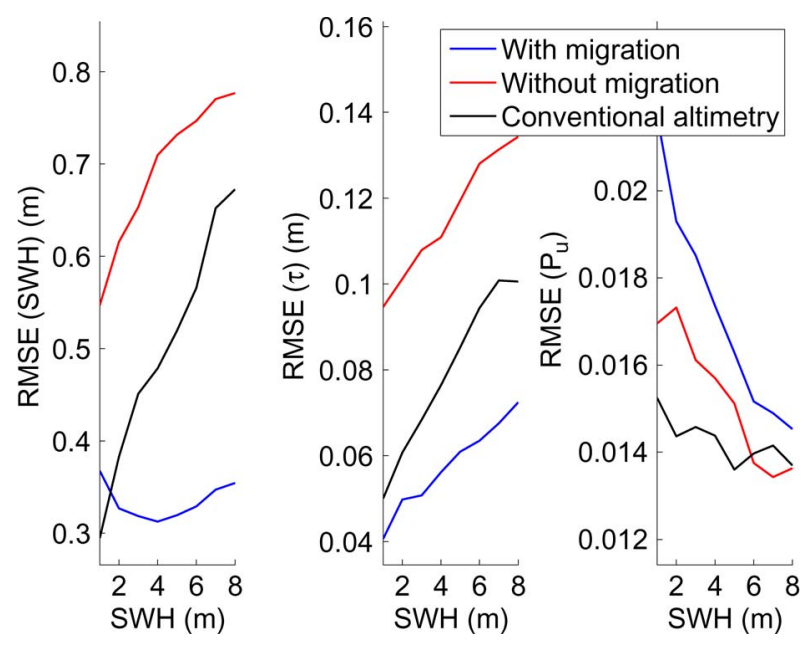

Fig. 12. Parameter RMSEs versus SWH (with $P_{u}=1$ and $\tau=31$ gates) for migrated and nonmigrated DD and conventional echoes (resulting from the average of 1000 Monte Carlo realizations).
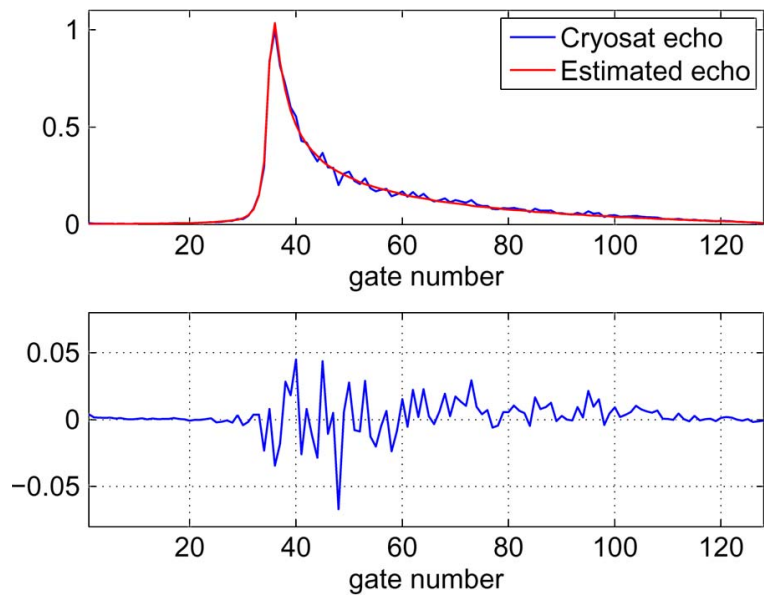

Fig. 13. Estimated Cryosat-2 echo using the proposed model. (Top) Real Cryosat-2 echo superimposed with its estimation; (bottom) difference between the real Cryosat- 2 echo and its estimation.

\section{RESUlTS FOR CRYOSAT-2 WAVEFORMS}

This section is devoted to the validation of the proposed semi-analytical model for oceanic Cryosat- 2 waveforms. The considered waveforms were obtained in August 2011 (the estimation was applied to the whole month of data) and were provided by the Cryosat- 2 processing prototype developed by the Centre National d'Études Spatiales which is doing the level 1 processing and particularly the Doppler, range migration, and multi-looking processings [38].

Fig. 13 (top) shows an example of estimated Cryosat- 2 echo using the proposed model. This figure shows an excellent fit between this estimated echo and the observed Cryosat-2 waveform (particularly in the leading and trailing edges of the waveform). This result is confirmed in Fig. 13 (bottom), which shows the error (difference) between the two echoes. Note that the maximum difference between the real echo and its estimation is on the order of $10 \%$, which is a small value due to the presence of noise (the reader is invited to consult [23] for additional results obtained with other waveforms). Considering the estimated parameters, the good agreement between the 
TABLE II

COMPARISON BETWEEN THE ESTIMATED PARAMETERS OF CA-SARM AND DDA

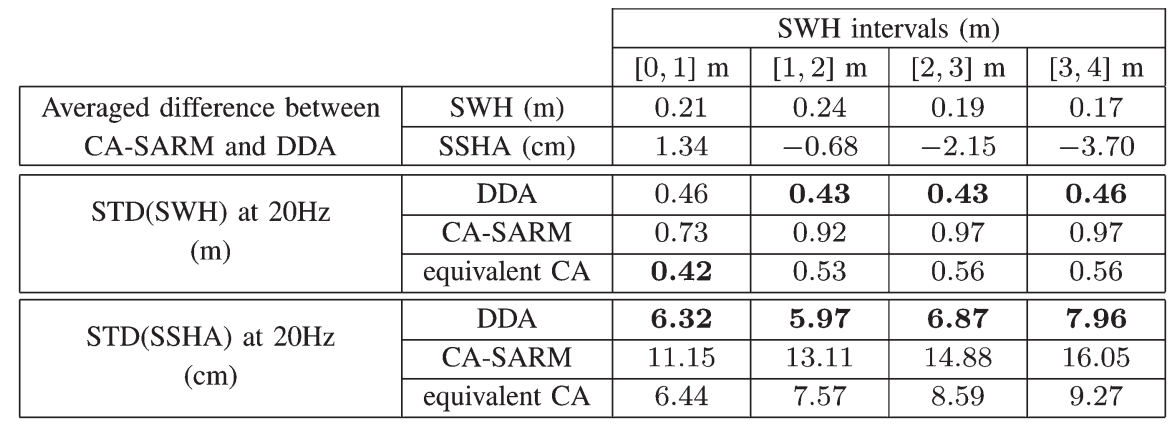
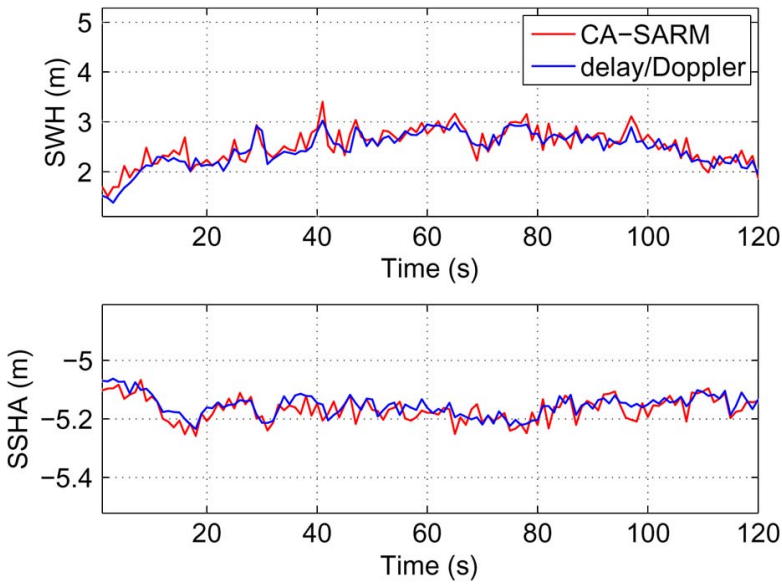

Fig. 14. Parameter estimates for 2 min of Cryosat-2 data for DD and CA-SARM echoes. (Top) SWH; (bottom) SSHA.
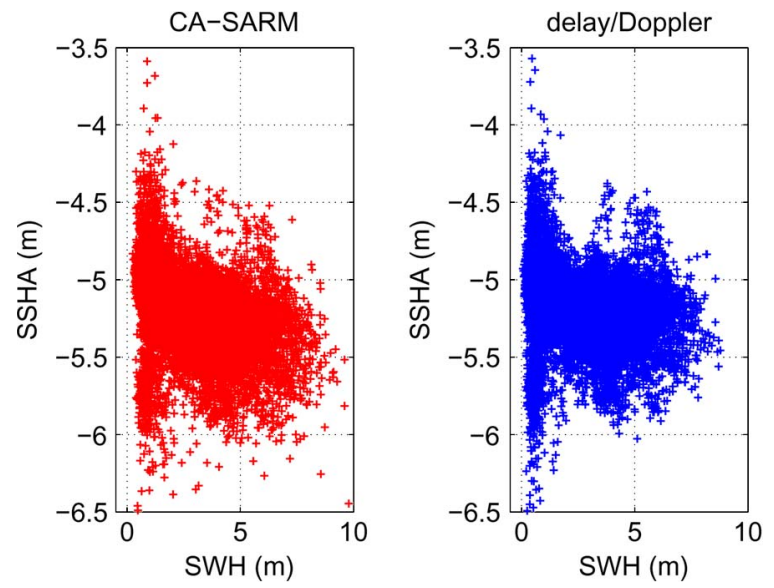

Fig. 15. Correlations between the estimated SWH and SSHA parameters for (left) CA-SARM and (right) DDA.

estimated DDA and CA-SARM parameters ${ }^{4}$ is illustrated in Fig. 14, which shows the estimated SWH and sea surface height anomaly (SSHA) parameters for 2 min of data (the SSHA is obtained by applying all environmental corrections on the estimated epoch). This agreement is confirmed in Fig. 15, showing similar correlations between the estimated SWH and SSHA using the two estimation procedures.

\footnotetext{
${ }^{4}$ The parameters of CA-SARM echoes have been estimated by the LS method described in Section III, where $s_{k}(\boldsymbol{\theta})=P\left(t_{k}\right)$ as defined in (1).
}

TABLE III

IMPROVEMENT FACTORS OF DDA WITH RESPECT TO CA

\begin{tabular}{|c|c|c|c|}
\cline { 2 - 4 } \multicolumn{1}{c|}{} & \multicolumn{3}{c|}{ Studies } \\
\cline { 2 - 4 } \multicolumn{1}{c|}{} & This paper & {$[40]$} & {$[42]$} \\
\hline SSHA & 1.26 & 1.18 & 1.43 \\
\hline SWH & 1.28 & 1.31 & 1.26 \\
\hline
\end{tabular}

Table II shows the averaged differences between the estimated parameters of CA-SARM and DDA. These results are represented for SWH $<4 \mathrm{~m}$ since more than $90 \%$ of the processed data satisfy this constraint. The differences between the CA-SARM and DDA estimations are very low. Table II also shows the averaged STDs ${ }^{5}$ for the parameters SWH and SSHA. These STDs have been obtained by considering groups of $m=20$ successive parameters [see (30)], i.e., one value of STD is obtained every second (the resulting STDs are known as $20-\mathrm{Hz}$ STDs). ${ }^{6}$ As expected, DDA provides lower STDs than CA-SARM, which is in agreement with the results in Section IV-C. Note that the equivalent CA STDs can be obtained by dividing the CA-SARM STDs by a factor of $\sqrt{3}$ as explained previously. This provides a good evaluation of DDA when compared to CA (used in the previous altimeters such as in Poseidon-3). The STD improvement can be evaluated by computing the ratio between the CA STDs and the DDA STDs (referred to as improvement factor in Table III). At $\mathrm{SWH}=2 \mathrm{~m}$, we obtain an SWH STD of $55 \mathrm{~cm}$ for CA and $43 \mathrm{~cm}$ for DDA, which shows an improvement factor of 1.28. Considering SSHA, we notice a CA STD of $8.16 \mathrm{~cm}$ and a DDA STD of $6.47 \mathrm{~cm}$, resulting in an improvement factor of about 1.26. Table III compares these improvement factors with results available in the literature. The obtained results are clearly in good agreement with those in [40] and [42] (the small differences are due to the fact that it is not possible to reproduce exactly the same simulation scenario).

Note finally that the STD results presented in Table II are similar to those obtained in the simulation (see Fig. 12). The improvement factors are also in agreement with those of simulated waveforms since we have obtained 1.24 for $\tau$ and 1.19 for $\mathrm{SWH}$ at $\mathrm{SWH}=2 \mathrm{~m}$. These similarities between simulated and real data results validate the proposed model.

\footnotetext{
${ }^{5}$ The averaged STDs have been obtained by averaging the obtained STDs for each $1-\mathrm{m}$ interval of SWH.

${ }^{6} \mathrm{The} 1-\mathrm{Hz}$ STDs can be deduced from the $20-\mathrm{Hz}$ STDs by dividing all results by the factor $\sqrt{20}$.
} 


\section{CONCLUSION}

This paper has defined a new semi-analytical model for DDA. A geometrical approach was used for computing an analytical expression of the FSIR. The analytical expression was obtained under the assumptions of a circular antenna pattern, no mispointing, no vertical speed effect, and a uniform scattering. This analytical expression was convolved with the PDF of the heights of the specular scatterers and the PTR of the radar, leading to the mean power of a DD altimetric waveform. An LS approach based on the Levenberg-Marquardt algorithm was then proposed to estimate the parameters of DD altimetric echoes. Simulation results performed on simulated data showed the good potential of DDA when compared to CA in terms of error reduction. The analysis of real Cryosat-2 waveforms confirmed the good performance of the proposed DD model. Extending the results obtained in this paper to a model including the mispointing angles is an interesting issue. This generalization might reduce the noise level since the Cryosat-2 echoes are known to present a mispointing of about $0.1^{\circ}$ in across-track and along-track directions [43]. The consideration of the antenna ellipticity, the satellite vertical speed effect, and a nonconstant backscattering coefficient might also improve the obtained performance. Finally, studying correlations between the estimated parameters is also an important issue that would deserve some attention.

\section{ACKNOWLEDGMENT}

The authors would like to thank T. Moreau, from Collecte Localisation Satellites, Ramonville-Saint-Agne, France, for the interesting discussions regarding the validation of the proposed model. The authors would also like to thank N. Picot, from the Centre National d'Études Spatiales, Toulouse, France, for his valuable suggestions that helped to improve this work.

\section{REFERENCES}

[1] G. Brown, "The average impulse response of a rough surface and its applications," IEEE Trans. Antennas Propag., vol. 25, no. 1, pp. 67-74, Jan. 1977.

[2] G. Hayne, "Radar altimeter mean return waveforms from near-normalincidence ocean surface scattering," IEEE Trans. Antennas Propag., vol. 28, no. 5, pp. 687-692, Sep. 1980.

[3] G. Brown, "A useful approximation for the flat surface impulse response," IEEE Trans. Antennas Propag., vol. 37, no. 6, pp. 764-767, Jun. 1989.

[4] G. Alberti, L. Festa, C. Papa, and G. Vingione, "A waveform model for near-nadir radar altimetry applied to the Cassini mission to Titan," IEEE Trans. Geosci. Remote Sens., vol. 47, no. 7, pp. 2252-2261, Jul. 2009.

[5] L. Amarouche, P. Thibaut, O. Z. Zanife, J.-P. Dumont, P. Vincent, and N. Steunou, "Improving the Jason-1 ground retracking to better account for attitude effects," Mar. Geod., vol. 27, no. 1/2, pp. 171-197, Aug. 2004.

[6] E. Rodriguez, "Altimetry for non-Gaussian oceans: Height biases and estimation of parameters," J. Geophys. Res., vol. 93, no. C11, pp. 14 10714 120, Nov. 1988.

[7] L. Amarouche, E. Thouvenot, B. Chapron, and O.-Z. Zanife, "A new estimator of the sea state bias using a three frequency radar altimeter," in Proc. IEEE IGARSS, Sydney, Australia, Jul. 9-13, 2001, pp. 2510-2512.

[8] J. Gómez-Enri, S. Vignudelli, G. D. Quartly, C. P. Gommenginger, P. Cipollini, P. G. Challenor, and J. Benveniste, "Modeling Envisat RA-2 waveforms in the coastal zone: Case study of calm water contamination," IEEE Geosci. Remote Sens. Lett., vol. 7, no. 3, pp. 474-478, Jul. 2010.

[9] A. Halimi, C. Mailhes, J.-Y. Tourneret, and P. Thibaut, "A new model for peaky altimetric waveforms," in Proc. IEEE IGARSS, Vancouver, BC, Canada, Jul. 24-29, 2011, pp. 2825-2828.
[10] A. Halimi, C. Mailhes, J.-Y. Tourneret, P. Thibaut, and F. Boy, "Parameter estimation for peaky altimetric waveforms," IEEE Trans. Geosci. Remote Sens., vol. 51, no. 3, pp. 1568-1577, Mar. 2013.

[11] C. Gommenginger, P. Thibaut, L. Fenoglio-Marc, G. Quartly, X. Deng, J. Gomez-Enri, P. Challenor, and Y. Gao, "Retracking altimeter waveforms near the coasts," in Coastal Altimetry, S. Vignudelli, A. G. Kostianoy, P. Cipollini, and J. Benveniste, Eds. Berlin, Germany: Springer-Verlag, 2011, pp. 61-101.

[12] R. K. Raney, "The delay/Doppler radar altimeter," IEEE Trans. Geosci. Remote Sens., vol. 36, no. 5, pp. 1578-1588, Sep. 1998.

[13] D. Wingham, C. R. Francis, S. Baker, C. Bouzinac, D. Brockley, R. Cullen, P. de Chateau-Thierry, S. Laxon, U. Mallow, C. Mavrocordatos, L. Phalippou, G. Ratier, L. Rey, F. Rostan, P. Viau, and D. Wallis, "Cryosat: A mission to determine the fluctuations in Earth's land and marine ice fields," Adv. Space Res., vol. 37, no. 4, pp. 841-871, Jul. 2006.

[14] L. Phalippou and V. Enjolras, "Re-tracking of SAR altimeter ocean powerwaveforms and related accuracies of the retrieved sea surface height, significant wave height and wind speed," in Proc. IEEE IGARSS, Barcelona, Spain, Jul. 23-27, 2007, pp. 3533-3536.

[15] Y. Shuang-Bao, L. He-Guang, X. Ke, and X. Xi-Yu, "The mean echo model and data process of SAR altimeter," in Proc. IEEE IGARSS, Vancouver, BC, Canada, Jul. 24-29, 2011, pp. 2077-2080.

[16] C. Martin-Puig, G. Ruffini, J. Marquez, D. Cotton, M. Srokosz, P. Challenor, R. K. Raney, and J. Benveniste, "Theoretical model of SAR altimeter over water surfaces," in Proc. IEEE IGARSS, Boston, MA, USA, Jul. 7-11, 2008, pp. 242-245.

[17] C. Gommenginger, C. Martin-Puig, S. Dinardo, D. Cotton, and J. Benveniste, "Improved altimetric performance of Cryosat-2 SAR mode over the open ocean and the coastal zone," presented at the Proc. IEEE IGARSS, Munich, Germany, Jul. 22-27, 2012, WE2.9.2.

[18] J.-P. Dumont, "Estimation optimale des paramètres altimétriques des signaux radar Poséidon,” Ph.D. dissertation, INP Toulouse, Toulouse, France, 1985

[19] S. Maus, C. M. Green, and J. D. Fairhead, "Improved ocean-geoid resolution from retracked ERS-1 satellite altimeter waveforms," Geophys. J. Int., vol. 134, no. 1, pp. 243-253, Feb. 1998.

[20] D. T. Sandwell and W. H. F. Smith, "Retracking ERS-1 altimeter waveforms for optimal gravity field recovery," Geophys. J. Int., vol. 163, no. 1, pp. 79-89, Oct. 2005.

[21] C. Martin-Puig and G. Ruffini, "SAR altimeter retracker performance bound over water surfaces," in Proc. IEEE IGARSS, Cape Town, South Africa, Jul. 12-17, 2009, pp. 449-452.

[22] L. Phalippou and F. Demeestere, "Optimal re-tracking of SAR altimeter echoes over open ocean: From theory to results for SIRAL2," in Ocean Surf. Topogr. Sci. Team Meet., San Diego, CA, USA, Oct. 2011, pp. $1-18$.

[23] A. Halimi, C. Mailhes, J.-Y. Tourneret, P. Thibaut, and F. Boy, A semianalytical model for delay/Doppler altimetry and its estimation algorithm, Univ. Toulouse-CLS-CNES, Toulouse, France, Tech. Rep., Jul. 2012. [Online]. Available: http://halimi.perso.enseeiht.fr/publications.html

[24] G. Picardi, R. Seu, and S. Sorge, "Extensive non-coherent averaging in Doppler beam sharpened space-borne radar altimeters," in Proc. IEEE IGARSS, Seattle, WA, USA, Jul. 6-10, 1998, pp. 2643-2645.

[25] D. B. Chelton, "Pulse compression and sea level tracking in satellite altimetry," J. Atmos. Oceanic Technol., vol. 6, no. 3, pp. 407-438, Jun. 1989.

[26] J. MacArthur, "Design of the Seasat-A radar altimeter," in Proc. OCEANS, Washington, DC, USA, Sep. 13-15, 1976, pp. 222-229.

[27] C. Martin-Puig, P. Berry, R. Smith, C. Gommenginger, G. Ruffini, P. Cipollini, L. Stenseng, O. Anderssen, D. Cotton, J. Benveniste, and S. Dinardo, "SAR altimetry over water surfaces," in Proc. Oceans Space, Venice, Italy, Apr. 2010, pp. 161-162.

[28] R. Moore and C. Williams, "Radar terrain return at near-vertical incidence," Proc. IRE, vol. 45, no. 2, pp. 228-238, Feb. 1957.

[29] D. Wingham, L. Phalippou, C. Mavrocordatos, and D. Wallis, "The mean echo and echo cross product from a beamforming interferometric altimeter and their application to elevation measurement," IEEE Trans. Geosci. Remote Sens., vol. 42, no. 10, pp. 2305-2323, Oct. 2004.

[30] R. K. Raney, "Cryosat SAR-mode looks revisited," IEEE Geosci. Remote Sens. Lett., vol. 9, no. 3, pp. 393-397, May 2012.

[31] R. K. Raney, "Maximizing the intrinsic precision of radar altimetric measurements," presented at the 20 Years Progress Radar Altimetry, Venice, Italy, Sep. 2012.

[32] A. Papoulis and S. U. Pillai, Probability, Random Variables and Stochastic Processes. New York, NY, USA: McGraw-Hill, 2002.

[33] D. P. Bertsekas, Nonlinear Programming. Belmont, MA: Athena Scientific, 1995. 
[34] J. Nocedal and S. Wright, Numerical Optimization. New-York, NY, USA: Springer-Verlag, 1999.

[35] J. Jensen and R. Raney, "Delay/Doppler radar altimeter: Better measurement precision," in Proc. IEEE IGARSS, Seattle, WA, USA, Jul. 6-10, 1998, pp. 2011-2013.

[36] W. H. F. Smith and R. Scharroo, "Pulse-to-pulse correlation in Cryosat SAR echoes from ocean surfaces: Implications for optimal pseudo-LRM waveform averaging," in Proc. Ocean Surf. Topogr. Sci. Team Meet., Venice, Italy, Sep. 2012, pp. 1-16.

[37] C. Gommenginger, P. Cipollini, D. Cotton, S. Dinardo, and J. Benveniste, "Finer, better, closer: Advanced capabilities of SAR altimetry in the open ocean and the coastal zone," in Proc. Ocean Surf. Topogr. Sci. Team Meet., Venice, Italy, Sep. 2012, pp. 1-24.

[38] F. Boy, J.-D. Desjonqueres, A. Halimi, P. Thibaut, T. Moreau, and N. Picot, "Cryosat processing prototype, LRM and SAR processing on CNES side and a comparison to DUACS SLA," in Proc. Ocean Surf. Topogr. Sci. Team Meet., Venice, Italy, Sep. 2012.

[39] J. D. Desjonqueres, G. Carayon, N. Steunou, and J. Lambin, "Poseidon-3 radar altimeter: New modes and in-flight performances," Mar. Geod., vol. 33, pp. 53-79, Aug. 2010, sup1.

[40] K. Giles, D. Wingham, N. Galin, R. Cullen, and W. Smith, "Precise estimates of ocean surface parameters from Cryosat," in Proc. Ocean Surf. Topogr. Sci. Team Meet., Venice, Italy, Sep. 2012, pp. 1-13.

[41] E. J. Walsh, "Pulse-to-pulse correlation in satellite radar altimeters," Radio Sci., vol. 17, no. 4, pp. 786-800, Aug. 1982.

[42] C. Gommenginger, C. Martin-Puig, S. Dinardo, D. Cotton, M. Srokosz, and J. Benveniste, "Improved altimetric accuracy of SAR altimeters over the ocean: Observational evidence from Cryosat-2 SAR and Jason-2," in Proc. Ocean Surf. Topogr. Sci. Team Meet., San Diego, CA, USA, Oct. 2011, pp. 1-35.

[43] W. H. F. Smith and R. Scharroo, "Retracking range, SWH, sigmanaught, and attitude in Cryosat conventional ocean data," in Proc. Ocean Surf. Topogr. Sci. Team Meet., San Diego, CA, USA, Oct. 2011, pp. 1-27.

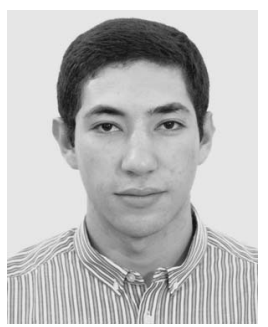

Abderrahim Halimi (S'11) received the Eng. degree in electronics from the National Polytechnic School of Algiers, Algiers, Algeria, in 2009 and the M.Sc. degree in signal processing from the Institut National Polytechnique de Toulouse, Toulouse, France, in 2010 . He is currently working toward the Ph.D. degree in the Signal and Communications Group, Institut de Recherche en Informatique de Toulouse (UMR 5505), University of Toulouse, Toulouse.

His research interests are centered around hyperspectral imaging and altimetry.

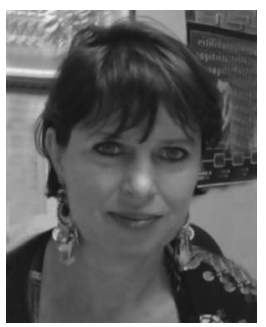

Corinne Mailhes (M'87) received the Ph.D. degree in signal processing from the University of Toulouse, Toulouse, France, in 1990.

She is currently a Professor with the University of Toulouse, a Member of the Institut de Recherche en Informatique de Toulouse (UMR 5505), and the Director of the Telecommunications for Space and Aeronautics laboratory. Her research interests include statistical signal processing, with particular interests in spectral analysis, parameter estimation, and biomedical signal processing.

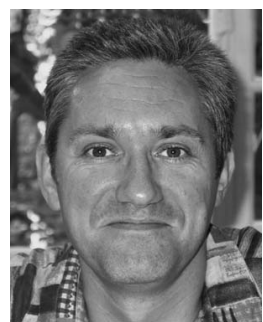

Jean-Yves Tourneret (SM'08) received the Eng. degree in electrical engineering from the École Nationale Supérieure d'Électronique, d'Électrotechnique, d'Informatique, d'Hydraulique et des Télécommunications (ENSEEIHT), Institut National Polytechnique de Toulouse, Toulouse, France, in 1989 and the Ph.D. degree from the Institut National Polytechnique de Toulouse in 1992.

$\mathrm{He}$ is currently a Professor with the University of Toulouse (ENSEEIHT), Toulouse, and a member of the Institut de Recherche en Informatique de Toulouse (UMR 5505). He has been serving as an Associate Editor for Signal Processing (since July 2013). His research activities are centered around statistical signal and image processing with a particular interest in Bayesian and Markov chain Monte Carlo methods.

Dr. Tourneret has been involved in the organization of several conferences, including the 2002 European Signal Processing Conference (Program Chair), the 2006 IEEE International Conference on Acoustics, Speech, and Signal Processing (plenaries), the 2012 IEEE Statistical Signal Processing Workshop (international liaisons), the 2013 IEEE International Workshop on Computational Advances in Multi-Sensor Adaptive Processing (local arrangement), and the 2014 IEEE Statistical Signal Processing Workshop (special sessions). He has been the General Chair of the Centre International de Mathématiques et Informatique de Toulouse workshop on optimization and statistics in image processing held in Toulouse in 2013. He has been a member of different technical committees, including the Signal Processing Theory and Methods Committee of the IEEE Signal Processing Society (2001-2007 and 2010-present). He served as an Associate Editor for the IEEE TRANSACTIONS ON SignaL PROCESSING (2008-2011).

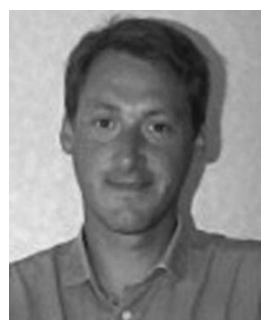

Pierre Thibaut received the Ph.D. degree in electronics and signal processing from the University of Toulouse, Toulouse, France, in 1994.

In 1996, he joined the Oceanography Division, Collecte Localisation Satellites (CLS), RamonvilleSaint-Agne, France, where he has been involved in many studies and projects aiming at defining, developing, and improving the scientific processing of altimeter missions such as Poseidon-1, Jason-1/ Jason-2, Envisat/RA-2, and Cryosat. Since 2010, he has been the Head of the Instruments and Physical Measurements Department, CLS, which is focused on altimetry and radiometry activities. He is a Jason-1 and Saral/AltiKa Coinvestigator and a Jason-2 Principal Investigator.

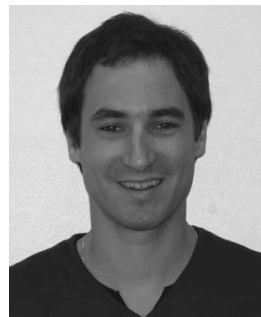

Francois Boy received the Eng. degree in physics from the École Centrale de Marseille, Marseille, France, in 2005 and the M.Sc. degree in electronics from the University of Toulouse, Toulouse, France, in 2006.

$\mathrm{He}$ is currently with the Altimetry and Radar Department, Centre National d'Études Spatiales, Toulouse. His research activities are centered around processing methods for synthetic aperture radar/Doppler altimetry systems (Cryosat and Sentinel-3 missions). 